\title{
A novel loss-of-function mutation of GATA3 (p.R299Q) in a Japanese family with Hypoparathyroidism, Deafness, and Renal Dysplasia (HDR) syndrome
}

\author{
Tetsuji Okawa ${ }^{1}$, Masanori Yoshida ${ }^{1 *}$, Takeshi Usui ${ }^{2}$, Takahiro Kudou ${ }^{3}$, Yasumasa Iwasaki ${ }^{4}$, Kazuki Fukuoka ${ }^{1}$,
} Norio Takahashi ${ }^{1}$, Yuka Uehara ${ }^{1}$ and Yutaka Oiso ${ }^{5}$

\begin{abstract}
Background: Hypoparathyroidism, deafness, and renal dysplasia (HDR) syndrome is a rare autosomal dominant disorder caused by mutations in the zinc finger transcription factor gene, GATA3. GATA3 has 2 zinc finger domains, which play an important role in the increase in target gene transcription activity.

Case presentation: A 50-year-old woman and her 27-year-old daughter were followed up because of hypoparathyroidism. They had bilateral sensorineural deafness. Abdominal computed tomography scanning revealed renal dysplasia in the mother, but no renal anomaly in the daughter. Direct sequencing of GATA3 gene revealed a novel heterozygous missense mutation at codon 299 (p.R299Q) in exon 4. This mutation is located at the junction between the 2 zinc fingers. The structure prediction showed that it caused a conformation change in this junction area, affecting the spatial position of the zinc fingers. Additionally, a more marked conformation change was observed in the N-terminal zinc finger region compared to that in the C-terminal region. Functional analysis of this mutant protein using an in vitro luciferase reporter assay system confirmed that the mutation abolished the enhancing effects of wild-type GATA3 on the promoter activity of the consensus GATA responsive element and that of human PTH gene.
\end{abstract}

Conclusion: We identified a novel R299Q mutation in GATA3 in a Japanese family with HDR syndrome. We confirmed that R299Q is a loss-of-function mutation, due to the extensive conformational change in the zinc fingers of GATA3.

Keywords: Hypoparathyroidism, Deafness, and renal dysplasia (HDR) syndrome, Zinc finger, GATA3, Heterozygous missense mutation, Structure prediction model, Luciferase reporter assay

\section{Background}

Hypoparathyroidism, deafness, and renal dysplasia (HDR) syndrome is a rare hereditary autosomal dominant disorder [1]. Haploinsufficiency of GATA3 gene, located on chromosome 10p15, is responsible for HDR syndrome $[2,3]$. Human GATA3 consists of 6 exons that span $20 \mathrm{~kb}$ of genomic DNA and encode 444 amino acids [4]. GATA3 is a dual zinc finger transcription factor that binds to the consensus motif $(\mathrm{A} / \mathrm{T}) \mathrm{GATA}(\mathrm{A} / \mathrm{G})$ on

\footnotetext{
*Correspondence: yoshidaz@ekisai.or.jp

'Department of Endocrinology and Diabetes, Nagoya Ekisaikai Hospital, 4-66 Shounen-cho, Nakagawawa-ku, Nagoya 454-8502, Japan

Full list of author information is available at the end of the article
}

the target gene promoter region [5-7]. This transcription factor plays a pivotal role in differentiation in the parathyroid gland, inner ear, kidney, thymus, and central nervous system $[8,9]$. Patients with HDR syndrome mainly present with a triad of clinical symptoms, viz., primary hypoparathyroidism, sensorineural deafness, and renal dysplasia with variable presentation even within individuals with the same mutation $[10,11]$. On the other hand, GATA3 also serves as a master regulator of the differentiation of $\mathrm{T}$ helper type 2 cells $[5,6]$, but no immune deficient symptom has been reported in patients with HDR syndrome.

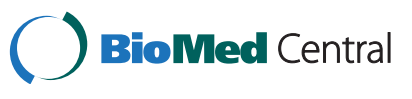

(c) 2015 Okawa et al. Open Access This article is distributed under the terms of the Creative Commons Attribution 4.0 International License (http://creativecommons.org/licenses/by/4.0/), which permits unrestricted use, distribution, and reproduction in any medium, provided you give appropriate credit to the original author(s) and the source, provide a link to the Creative Commons license, and indicate if changes were made. The Creative Commons Public Domain Dedication waiver (http://creativecommons.org/publicdomain/zero/1.0/) applies to the data made available in this article, unless otherwise stated. 
Here, we report a novel mutation of GATA3 in a Japanese family with HDR syndrome, presenting with different renal phenotypes. We analyzed the 3-dimensional structure and transcriptional function of this mutant GATA3.

\section{Case presentation}

A 50-year-old Japanese woman had been followed up at our hospital because of hypoparathyroidism. At the age of 33 years, the patient had suddenly experienced a general convulsion with loss of consciousness and was taken to the emergency room of our hospital. Upon arrival, she regained consciousness. She was positive for the Trousseau sign. Blood examination showed severe hypocalcemia $(5.2 \mathrm{mg} / \mathrm{dL})$ and hyperphosphatemia $(4.7 \mathrm{mg} /$ $\mathrm{dL})$ with a relatively low level of intact PTH $(7 \mathrm{pg} / \mathrm{mL})$. Serum creatinine and blood urea nitrogen levels were normal $(0.6 \mathrm{mg} / \mathrm{dL}$ and $9.1 \mathrm{mg} / \mathrm{mL}$, respectively) with no other electrolyte disorder. Percentage of tubular reabsorption of phosphate was 98.4 \% (reference range: 81-90\%). Anterior pituitary hormone levels and thyroid and adrenal functions were normal. She was diagnosed with idiopathic hypoparathyroidism, and calcium lactate and $1 \alpha$-hydroxyvitamin $\mathrm{D}_{3}$ treatment were initiated. After calcium lactate was discontinued, administration of $2 \mu \mathrm{g}$ of $1 \alpha$-hydroxyvitamin $\mathrm{D}_{3}$ was sufficient to maintain her serum calcium level within the normal range. Head computed tomography (CT) scanning showed severe brain calcification. CT images at the age of 47 years are shown in Fig. 1a and b; calcification was observed in the bilateral basal ganglia, thalamus, cerebellum, and deep white matter. She had mild juvenile cataracts in both eyes. Fluorescence in situ hybridization did not indicate $22 \mathrm{q} 11.2$ syndrome, as no microdeletion was observed in the $22 \mathrm{q} 11.2$ region (data not shown). Hearing disturbance progressed gradually; an audiogram performed at the age of 41 years indicated bilateral sensorineural deafness (right: $47 \mathrm{~dB}$, left: $55 \mathrm{~dB}$ ). Abdominal CT revealed renal dysplasia on the right side (Fig. 1c). These clinical findings were consistent with HDR syndrome.

This patient's 27-year-old daughter also had hypoparathyroidism, which was incidentally identified when she visited our hospital for a common cold at the age of 14 years; blood examination showed hypocalcemia $(5.7 \mathrm{mg} / \mathrm{dL})$ and hyperphosphatemia $(6.8 \mathrm{mg} / \mathrm{dL})$ with a low level of intact PTH $(9 \mathrm{pg} / \mathrm{mL})$. Thereafter, she had received $1 \mu \mathrm{g}$ of $1 \alpha-$ hydroxyvitamin $D_{3}$. She had a moderate hearing disturbance (right: $56 \mathrm{~dB}$, left: $47 \mathrm{~dB}$ ). Unlike her mother, faint calcifications in the basal ganglia and no renal abnormalities were observed.

Sequencing analysis was performed for all coding regions, including the adjacent intronic regions, of GATA3 from the peripheral blood of the mother. A novel heterozygous p.R299Q (CGG > CAG) missense mutation was found in exon 4 (Fig. 2). This codon 299 amino acid of

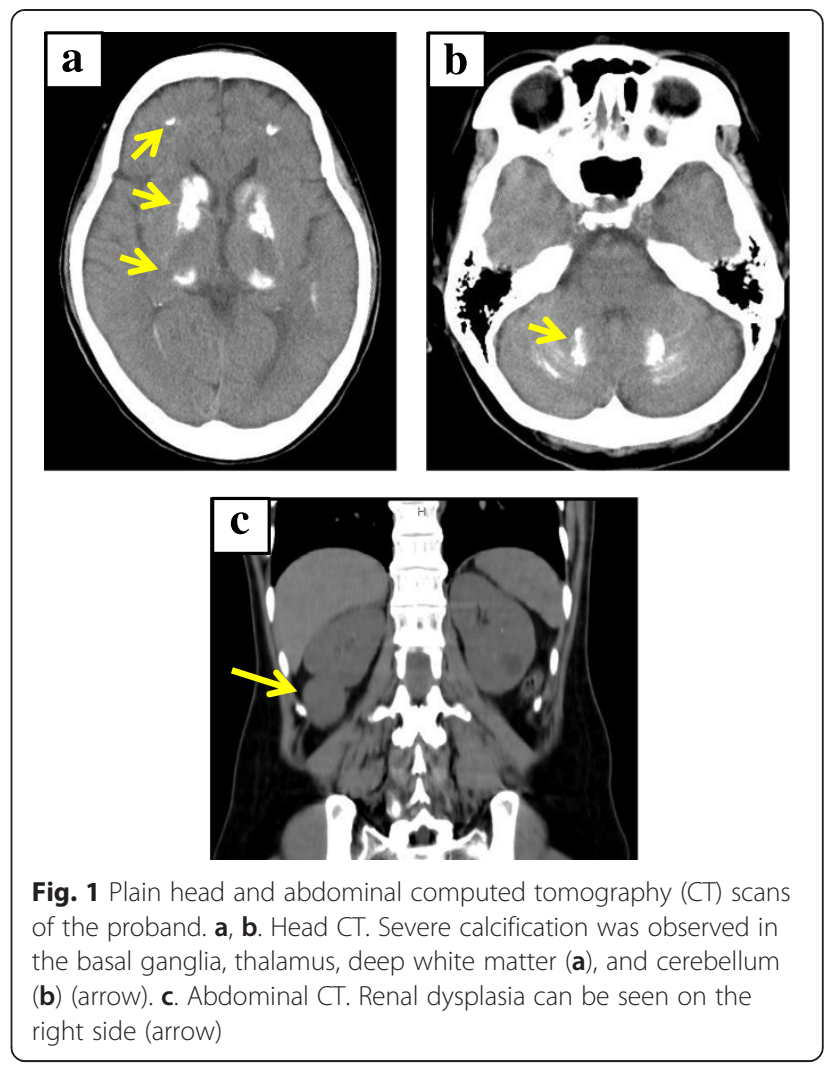

GATA3 was located in the liker region, consisting of 30 amino acids, between 2 consecutive zinc finger domains. These zinc fingers are important for the increase in the transcriptional activity of GATA3 target genes.

We analyzed the 3-dimensional structural models of wild-type (GATA3/WT) and mutant (GATA3/R299Q) GATA3 using the Sequence to Function Annotation Server (SFAS) provided by the Protein Data Bank Japan (PDBj: http://pdbj.org/) [12]. The structure of both proteins was compared and visualized by UCSF Chimera, developed by the Resource for Biocomputing, Visualization, and Informatics (RBVI) (http://www.cgl.ucsf.edu/chimera/) [13]. The protein conformation showing the 2 zinc fingers is presented in Fig. 3. This mutation altered the spatial position of the 2 zinc fingers through a conformation change in the linker between the zinc fingers. A more marked conformational change was observed in the position of the $\mathrm{N}$ terminal zinc finger ( $\mathrm{ZnF} 1$; residues 264-288) than in the C-terminal zinc finger ( $\mathrm{ZnF} 2$; residues 318-342), probably because the Arg-299 residue is located near the zinc ion of ZnF1 (Fig. 3a). The conformational change also extended into a part of ZnF2. This extensive conformational change probably affects the biological function of GATA3.

The transcriptional function of GATA3/R299Q was evaluated using a luciferase reporter assay system. The transcription activity of pGATA/Luc with a consensus 


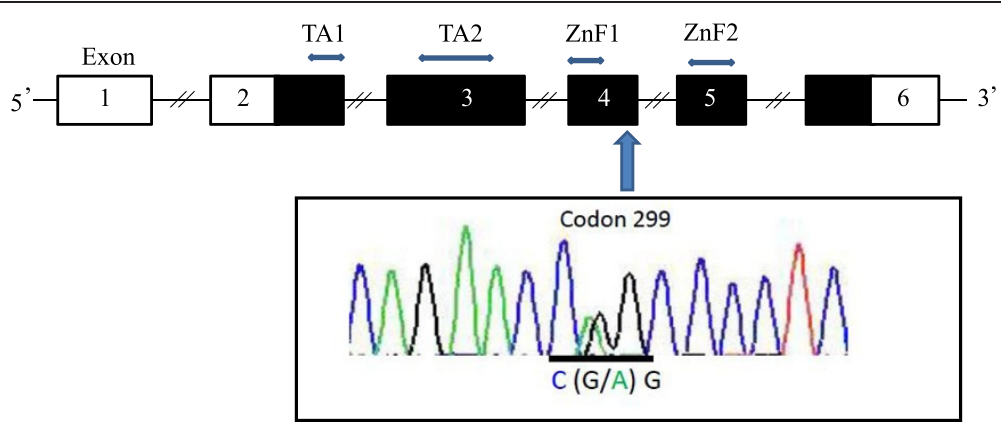

Fig. 2 Direct sequencing of GATA3. Upper panel shows the genomic structure of GATA3, consisting of 6 exons (indicated by boxes). The black and white boxes denote the coding regions and the untranslated regions, respectively. GATA3 encodes a protein with 2 transactivating domains (TA1 and TA2) and 2 zinc finger domains ( $\mathrm{N}$-terminal zinc finger [ZnF1] and C-terminal zinc finger [ZnF2]). ZnF1 is encoded by exon 4 and ZnF2 is encoded by exon 5. The arrow indicates the mutation site. The lower panel shows the nucleotide sequences around codon 299 in exon 4 of GATA3

GATA responsive element significantly increased when the GATA3/WT expression vector was transfected, whereas this enhancing effect was completely lost in the GATA3/R299Q vector-introduced cells (Fig. 4a). This result indicated that GATA3/R299Q severely affected the transcription activity of various GATA3-regulated genes. We next examined the effects of GATA3/WT and GATA3/ R299Q on the promoter activity of the PTH gene. GATA3/ WT overexpression significantly enhanced the transcription activity of human $P T H$ in a dose-dependent manner, whereas this stimulatory effect was not observed with GATA3/R299Q overexpression (Fig. 4b). These data suggested that the GATA3/R299Q protein failed to increase the PTH gene expression. The extensive conformational change of GATA3/R299Q most likely reduces the transcriptional activity of GATA3 target genes associated with fetal differentiation of the organs affected in HDR syndrome, such as the parathyroid gland, inner ear, and kidney.

\section{Method}

\section{Preparation of DNA and sequence analysis}

DNA was extracted from peripheral blood leukocytes using standard methods. All PCR-amplified exons and splice junctions of GATA3 from the proband were directly sequenced [14], after permission was obtained from the patient and the ethical committees of Nagoya Ekisaikai Hospital and National Hospital Organization Kyoto Medical Center.

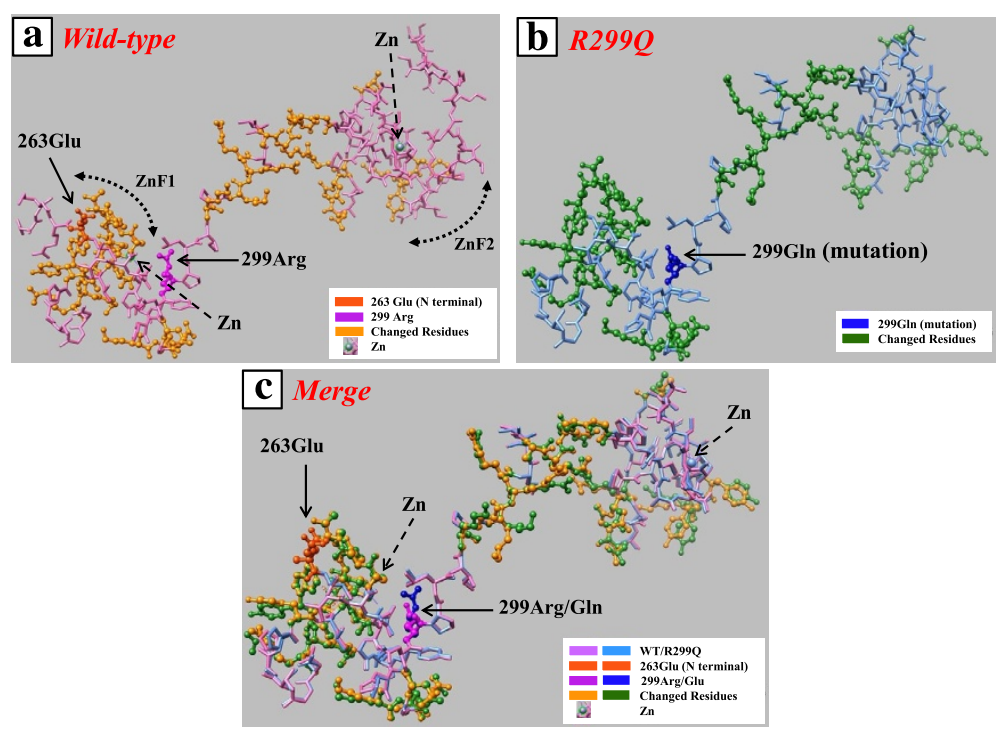

Fig. 3 Three-dimensional structural modeling of wild-type and mutant GATA3 proteins. a, Wild-type GATA3 protein. b, GATA3 mutant protein (R299Q). c, Merge of (a) and (b). Residues 263-347 are shown. This region includes ZnF1 (residues 264-288) and ZnF2 (residues 318-342). The arrows indicate residues 263 ( $\mathrm{N}$-terminal) and 299. Residues with a significant conformational change by R299Q mutation are color-coded (bright yellow [wild-type] and green [mutant]) 

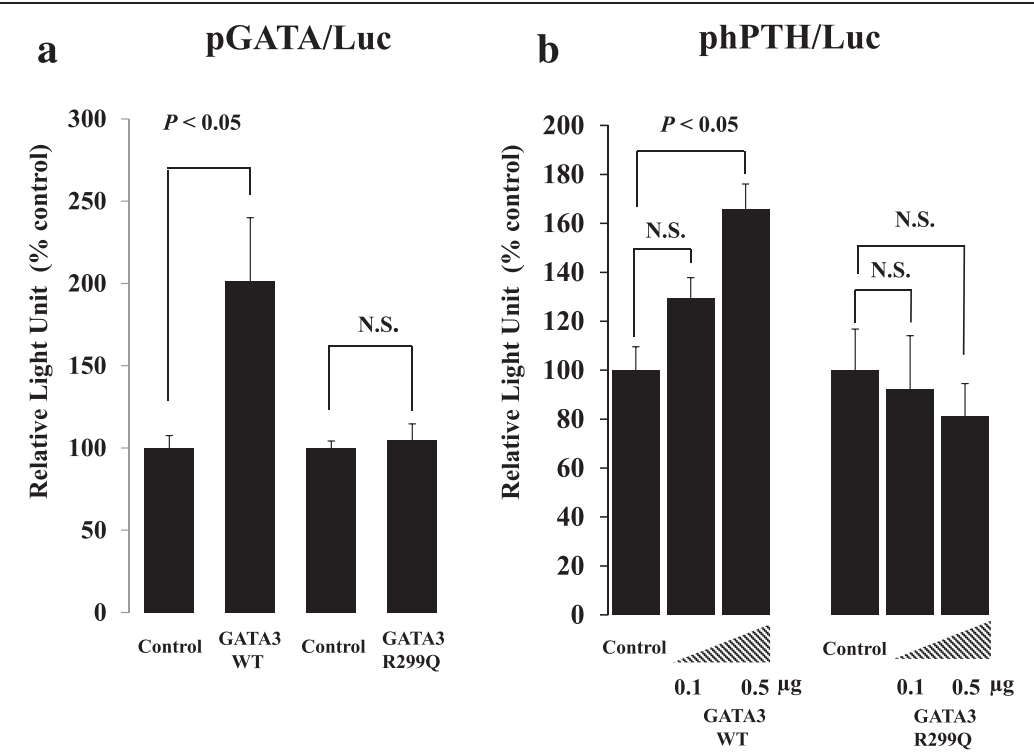

Fig. 4 Functional analysis of wild-type and mutant GATA3 proteins using a luciferase reporter assay system. a, Effects of the overexpression of wild-type and mutant GATA3 on the transcription activity of the heterologous promoter. Mutant GATA3 overexpression abolished the enhancing effects of wild-type GATA3 on the promoter containing the consensus GATA responsive element (pGATA/Luc). $\mathbf{b}$, Effects of the overexpression of wild-type and mutant GATA3 on human PTH gene expression. Wild-type GATA3 overexpression significantly enhanced the transcription activity of human PTH (phPTH/Luc), whereas this stimulatory effect was not observed with mutant GATA3 overexpression. Data are expressed as folds increase over control group. $P<0.05$ vs. corresponding control

\section{Plasmid construction}

Full-length human GATA3 cDNA was kindly provided by RIKEN BioResource Center (Tsukuba, Japan) as a plasmid [15]. A GATA3 expression vector (GATA3/WT) was constructed by insertion of the human GATA3 cDNA into a pRc/Rous sarcoma virus (RSV) vector (Invitrogen, San Diego, CA). Specific primers used for PCR were as follows: forward 5' - GAGGCCATGGAGGTGACGGC-3', reverse 5'-TCTAACCCATGGCGGTGACC-3'; mutant construct (GATA3/R299Q) was made from GATA3/WT by a sitedirected mutagenesis technique using the primers as follows: forward 5'-GGACAGAACCAGCCCCTCATTA-3', reverse 5 '-TAATGAGGGGCTGGTTCTGTCC-3'. For the luciferase assay, 2 luciferase reporter plasmids were used: pGATA/Luc, containing the consensus GATA binding element (LR-2103, Signosis, Santa Clara, CA), and phPTH/ Luc containing the $5^{\prime}$-promoter region of human $P T H$ $(-2000 /+54 ;+1$ designates the transcription start site) [16].

\section{Cell culture and transfection}

HepG2 cells were maintained in $\mathrm{T}_{75}$ culture flasks using DMEM culture medium supplemented with $10 \%$ (v/v) fetal bovine serum, penicillin $(100 \mathrm{U} / \mathrm{ml})$, and streptomycin $(100 \mu \mathrm{g} / \mathrm{ml})$ at $37{ }^{\circ} \mathrm{C}$ with $5 \% \mathrm{CO}_{2}$. In each experiment, cells were cultured in 24-well plates, and then transient transfection was carried out using FuGene 6 reagent (Promega, Madison, WI), $2 \mu \mathrm{l}$ of FuGene 6 was used per $1 \mu \mathrm{g}$ of plasmids (reporter plasmids: $0.5 \mu \mathrm{g}$; expression plasmids: $0.5 \mu \mathrm{g}$ ) for each well. When different doses of an expression plasmid were used in an experiment, the total amount of transfected plasmid DNA was kept constant by addition of the empty control vector (pRc/RSV). Cells were harvested $48 \mathrm{~h}$ after the transfection and a luciferase assay was performed as described previously [17].

\section{Computer modeling of GATA3 structure}

Three-dimensional structural modeling of wild-type and mutant GATA3 proteins was analyzed using SFAS provided by $\mathrm{PDBj}$ (http://pdbj.org/) [12]. The structural information of the wild-type GATA3 was derived from chain A of PDB entry 4hc7. Molecular graphics of the comparison of protein structures (residues 263-347) were created by UCSF Chimera which is a program to display molecular graphics of protein and nucleic acids developed by RBVI funded by the National Institutes of Health (http://www.cgl.ucsf.edu/chimera/) [13].

\section{Statistics}

Samples in each group of transcription activation experiments were analyzed in triplicate. All of the experiments were performed more than twice to confirm the reproducibility, and the representative data are shown in the results. Data are expressed as means \pm S.E. The differences between experimental values were analyzed by Student's $t$ test. Statistical analysis was performed using one-way ANOVA. $P$-values below 0.05 were considered significant. 


\section{Discussion}

In the present case, we identified a novel heterozygous missense mutation of GATA3 in a Japanese family affected with HDR syndrome. This R299Q mutation induced an orientation change of the 2 zinc fingers and abolished its physiological function as a transcription factor.

The clinical manifestation of HDR syndrome is heterogeneous [3]. A triad of HDR symptoms is observed in $62.3 \%$ of patients; $28.6 \%$ of patients show only hypoparathyroidism and deafness, and $2.6 \%$ of patients show only deafness and renal disease [18]. Hypoparathyroidism and hearing loss is the most common combination. Many different renal anomalies, with variable penetrance, are observed, including renal dysplasia, hypoplasia, aplasia, and vesico-ureteral reflux $[3,10,11]$. In addition, similar to our case, patients with HDR syndrome present with heterogeneous clinical manifestation even among individuals having the same mutation $[10,11]$. The mechanism of heterogeneous symptoms has not been fully elucidated. In animal study, Grote et al. showed that the nephric ductspecific inactivation of Gata3 leads to a wide spectrum of urogenital malformations through the glial cell linederived neurotrophic factor/Ret signaling pathway [19]. Gata3 prevents ectopic ureter budding and premature differentiation of nephric duct cells. On the other hand, Gata3, which is also expressed in spiral ganglion neurons throughout their development, is essential for formation of the intricately patterned connections in the cochlea [20].

GATA3 belongs to a family of dual zinc finger transcription factors. Zinc fingers of the mammalian GATA proteins (GATA 1-6) have a Cys-X2-Cys- $\times 17-C y s-X 2$-Cys structure (with $\mathrm{X}$ representing any amino acid residue), in which a single zinc ion is coordinated by 4 cysteine residues [7]. Its tertiary structure reveals 2 anti-parallel $\beta$-sheets, an $\alpha$ helix, and a long loop. The $\alpha$-helix binds into the major groove of DNA. In GATA3, ZnF2 is essential to the binding of the transcription factor to the consensus sequence (A/T)GATA(A/G) on the target gene promoter region, whereas $\mathrm{ZnF} 1$ is thought to stabilize this binding through its interaction with another cofactor, Friends of GATA (FOG) $2[21,22]$.

In this study, the 3-dimensional conformation of wildtype and mutant GATA3 was analyzed using SFAS, which runs several external programs for sequence alignment and structural modeling and organizes their results. Protein structure comparison was presented using another web application, UCSF Chimera, to construct high-resolution images. The structure of residues 263-347, ranging from $\mathrm{ZnF1}$ to $\mathrm{ZnF} 2$, is displayed in Fig. 3. The configuration of the linker between the zinc fingers was affected severely by the mutation R299Q, which is located between the 2 zinc fingers. In addition, the mutation created a greater conformational change in $\mathrm{ZnF1}$ than in $\mathrm{ZnF}$, probably because Arg-299 is located near the zinc ion of $\mathrm{ZnF1}$. This
Arg residue may stabilize a coordinate bond between the zinc ion and Cys residues, which are the crucial components of ZnF1. Substituting the basic amino acid (Arg) with neutral amino acid (Gln) may affect this stabilization and cause a change in the orientation in the protein structure. Furthermore, this conformational change spread into ZnF2. These results suggest that R299Q mutation impairs the function of both $\mathrm{ZnF} 1$ and $\mathrm{ZnF} 2$.

To date, the conformation of GATA3 mutant proteins has been investigated only in 3 cases of HDR syndrome $[10,11,23]$. Gaynor et al. showed that Thr272Ile, located in $\mathrm{ZnF1}$, resulted in the loss of a polar side chain interaction between Thr-272 and Leu-274 [23]. This mutation changed the $\mathrm{ZnF} 1$ structure, leading to loss of DNA binding and FOG2 interactions. Ali et al. also showed that Leu348Arg could alter the transcriptional activity of GATA3 because of the substitution of a non-polar hydrophobic Leu residue for a positively charged Arg residue; this Leu 348 residue is located in $\mathrm{ZnF2}$ and lies at the end of an $\alpha$-helix which makes contact with DNA at the DNA major groove within the GATA motif of the promoter region [10]. Moreover, Chiu et al. analyzed the conformation change caused by Arg353Ser, which is located at the C-terminal DNA-binding region following ZnF2 (residues 318-342), using NNPredict software. This mutation was predicted to disrupt the helix turn composed of residues 355-358, because of the substitution of a basic for a polar, uncharged residue, which changed the angle between $\mathrm{ZnF} 2$ and the adjacent $\mathrm{C}$ terminal tail [11]. However, our case is the first report of a conformation change caused by a mutation at the junction between the 2 zinc fingers; the amino acid substitution affected the conformation of both $\mathrm{Zn}$ fingers. Structure prediction model obtained by PDBj provides useful information about GATA3 mutant. Amino acids are classified into 4 groups such as nonpolar, polar, acidic, and basic amino acids. Substitution between amino acids belonging to different groups is likely to cause critical conformation change.

We also confirmed that R299Q was a loss-of-function mutation using an in vitro reporter assay system. GATA3/ R299Q overexpression failed to increase the transcription activity of the GATA responsive element. In addition, our data showed that GATA3/WT activated PTH transcriptional activity, whereas GATA3/R299Q did not (Fig. 4b). This result is of interest, as there has been no previous direct evidence that GATA3 is a positive regulator of $P T H$ expression. The consensus GATA3 binding element is not present in the $5^{\prime}$-promoter region of human PTH, suggesting that the observed enhancing effect of GATA3 is caused by an indirect pathway, rather than by the direct binding of GATA3. Further investigations are required to elucidate this molecular mechanism. Our results suggest that GATA3 plays an important role not only in the 
development of the parathyroid gland in fetus, but also in PTH regulation after birth.

\section{Conclusion}

In this study, we identified a novel heterozygous missense mutation of GATA3 in a Japanese family with HDR syndrome. We confirmed that the R299Q mutant is a loss-offunction mutation causing an extensive conformational change of 2 zinc fingers.

\section{Consent}

Written informed consent was obtained from both patients for publication of this case report and all accompanying images. A copy of the written consent is available for review by the Editor of this journal.

\section{Abbreviations}

CT: Computed tomography; GATA3: GATA binding protein 3; HDR: Hypoparathyroidism, deafness, and renal dysplasia; PTH: Parathyroid hormone.

\section{Competing interests}

The authors declare that they have no conflict of interest.

\section{Authors' contributions}

TO performed clinical management as a primary physician of the proband and drafted the initial manuscript. MY contributed to the conception and design of the work and edited and reviewed the manuscript. TU contributed to sequence analysis. TK performed structural analysis and proposed a useful suggestion to the results. Yl performed in vitro functional analysis and statistical analysis. KF performed clinical management as the primary physician to the proband's daughter. NT and UF participated in acquisition of the data. YO supervised manuscript presentration. All authors read and approved the final manuscript.

\section{Acknowledgment}

The authors thank RIKEN BioResource Center for providing the GATA3 expression vector. Molecular graphics and analyses were performed with the UCSF Chimera package. Chimera was developed by the Resource for Biocomputing, Visualization, and Informatics at the University of California, San Francisco (supported by NIGMS P41-GM103311). This work was supported in part by the grant-in-aid "Platform for drug discovery, informatics, and structural life sciences" from the Ministry of Education, Culture, Sports, Science and Technology (MEXT), Japan.

\section{Author details}

${ }^{1}$ Department of Endocrinology and Diabetes, Nagoya Ekisaikai Hospital, 4-66 Shounen-cho, Nakagawawa-ku, Nagoya 454-8502, Japan. ${ }^{2}$ Clinical Research Institute, National Hospital Organization Kyoto Medical Center, Kyoto 612-8555, Japan. ${ }^{3}$ Laboratory of Protein Informatics, Research Center for State-of-the-Art Functional Protein Analysis, Institute for Protein Research, Osaka University, Suita 565-0871, Japan. ${ }^{4}$ Health Care Center, Kochi University, Kochi 780-8520, Japan. ${ }^{5}$ Department of Endocrinology and Diabetes, Nagoya University Graduate School of Medicine, Nagoya 466-8550, Japan.

\section{Received: 19 May 2015 Accepted: 25 October 2015}

\section{Published online: 30 October 2015}

\section{References}

1. Bilous RW, Murty G, Parkinson DB, Thakker RV, Coulthard MG, Burn J, et al. Brief report: autosomal dominant familial hypoparathyroidism, sensorineural deafness, and renal dysplasia. N Engl J Med. 1992;327:1069-74.

2. Van Esch $H$, Groenen $P$, Nesbit MA, Schuffenhauer S, Lichtner $P$, Vanderlinden G, et al. GATA3 haplo-insufficiency causes human HDR syndrome. Nature. 2000:406:419-22.

3. Upadhyay J, Steenkamp DW, Milunsky JM. The syndrome of hypoparathyroidism, deafness, and renal anomalies. Endocr Pract. 2013;19:1035-42
4. Labastie MC, Bories D, Chabret C, Grégoire JM, Chrétien S, Roméo PH. Structure and expression of the human GATA3 gene. Genomics. 1994;21:1-6.

5. Marine J, Winoto A. The human enhancer-binding protein Gata3 binds to several T-cell receptor regulatory elements. Proc Natl Acad Sci U S A. 1991;88:7284-8.

6. Orkin SH. GATA-binding transcription factors in hematopoietic cells. Blood. 1992;80:575-81.

7. Yang Z, Gu L, Romeo PH, Bories D, Motohashi H, Yamamoto M, et al. Human GATA-3 trans-activation, DNA-binding, and nuclear localization activities are organized into distinct structural domains. Mol Cell Biol. 1994;14:2201-12

8. Debacker C, Catala M, Labastie MC. Embryonic expression of the human GATA-3 gene. Mech Dev. 1999:85:183-7

9. van Looij MA, Meijers-Heijboer H, Beetz R, Thakker RV, Christie PT, Feenstra LW, et al. Characteristics of hearing loss in HDR (hypoparathyroidism, sensorineural deafness, renal dysplasia) syndrome. Audiol Neurootol. 2006;11:373-9.

10. Ali A, Christie PT, Grigorieva IV, Harding B, Van Esch H, Ahmed SF, et al. Functional characterization of GATA3 mutations causing the hypoparathyroidism-deafness-renal (HDR) dysplasia syndrome: insight into mechanisms of DNA binding by the GATA3 transcription factor. Hum Mol Genet. 2007;16:265-75

11. Chiu WY, Chen HW, Chao HW, Yann LT, Tsai KS. Identification of three novel mutations in the GATA3 gene responsible for familial hypoparathyroidism and deafness in the Chinese population. J Clin Endocrinol Metab. 2006;91:4587-92

12. Kinjo AR, Suzuki $H$, Yamashita $R$, Ikegawa $Y$, Kudou $T$, Igarashi $R$, et al. Protein Data Bank Japan (PDBj): maintaining a structural data archive and resource description framework format. Nucleic Acids Res. 2012;40(Database issue):D453-60. doi:10.1093/nar/gkr811.

13. Pettersen EF, Goddard TD, Huang CC, Couch GS, Greenblatt DM, Meng EC, et al. UCSF Chimera-a visualization system for exploratory research and analysis. J Comput Chem. 2004;25:1605-12.

14. Nanba K, Usui T, Nakamura M, Toyota Y, Hirota K, Tamanaha T, et al. A novel GATA3 nonsense mutation in a newly diagnosed adult patient of hypoparathyroidism, deafness, and renal dysplasia (HDR) syndrome. Endocr Pract. 2013;19:e17-20.

15. Suzuki Y, Yoshitomo-Nakagawa K, Maruyama K, Suyama A, Sugano S. Construction and characterization of a full length-enriched and a 5'-end-enriched cDNA library. Gene. 1997;200:149-56.

16. Kawahara M, Iwasaki $Y$, Sakaguchi $K$, Taguchi T, Nishiyama M, Nigawara T, et al. Involvement of GCMB in the transcriptional regulation of the human parathyroid hormone gene in a parathyroid-derived cell line PT-r: effects of calcium and 1,25(OH)2D3. Bone. 2010;47:534-41.

17. Iwasaki Y, Oiso Y, Saito H, Majzoub JA. Positive and negative regulation of the rat vasopressin gene promoter. Endocrinology. 1997;138:5266-74.

18. Ferraris S, Del Monaco AG, Garelli E, Carando A, De Vito B, Pappi P, et al. HDR syndrome: a novel "de novo" mutation in GATA3 gene. Am J Med Genet A. 2009:149A:770-5.

19. Grote D, Boualia SK, Souabni A, Merkel C, Chi X, Costantini F, et al. Gata3 acts downstream of beta-catenin signaling to prevent ectopic metanephric kidney induction. PLoS Genet. 2008;4(12):e1000316. doi: 10.1371/journal.pgen.1000316.

20. Appler JM, Lu CC, Druckenbrod NR, Yu WM, Koundakjian EJ, Goodrich LV Gata3 is a critical regulator of cochlear wiring. J Neurosci. 2013;33:3679-91.

21. Nesbit MA, Bowl MR, Harding B, Ali A, Ayala A, Crowe C, et al. Characterization of GATA3 mutations in the hypoparathyroidism, deafness, and renal dysplasia (HDR) syndrome. J Biol Chem. 2004;279:22624-34.

22. Zahirieh A, Nesbit MA, Ali A, Wang K, He N, Stangou M, et al. Functional analysis of a novel GATA3 mutation in a family with the hypoparathyroidism, deafness, and renal dysplasia syndrome. J Clin Endocrinol Metab. 2005;90:2445-50.

23. Gaynor KU, Grigorieva IV, Nesbit MA, Cranston T, Gomes T, Gortner L, et al. A missense GATA3 mutation, Thr272lle, causes the hypoparathyroidism, deafness, and renal dysplasia syndrome. J Clin Endocrinol Metab. 2009;94:3897-904. 